\title{
Bullwhip Effect in a Supply Chain with Trend Demand: An Agent-based Simulation View
}

\author{
Hongsen Wang ${ }^{1 *}$ and Suling $\mathrm{Jia}^{2}$ \\ ${ }^{1,2}$ School of Economics and Management, Beihang University, Beijing, China \\ ${ }^{1}$ crifny@sina.com, ${ }^{2}$ jiasuling@buaa.edu.cn
}

\begin{abstract}
The bullwhip effect is one of the core problems in supply chain management. Research on it has made a great progress over the years. However, little research has discussed it combining trend demand with order-up-to level replenishment policy in a multi-echelon supply chain. The fluctuation of the bullwhip effect itself is also seldom analyzed. This paper studies the two problems from the view of agent-based simulation. An agent-based model with complex calculation is developed. One highlight is that the players in the supply chain dynamically and intelligently estimate corresponding parameters at every tick. The results indicate that both the bullwhip effect and its fluctuation aggravate with the increase of leadtime. The trend size of customer demand has little influence on the bullwhip effect. The noise of customer demand does not affect the bullwhip effect significantly when it accords with normal distribution. The mean of the bullwhip effect changes differently with the increase of noise autocorrelation coefficient when noise is an $A R(1)$ process. Meanwhile, the standard deviation of the bullwhip effect roughly changes in line with the mean. This paper also presents one bullwhip effect measurement suitable for trend demand.
\end{abstract}

Keywords: Bullwhip Effect; Multi-echelon Supply Chain; Trend Demand; Holt Forecasting Method; Agent-based Simulation

\section{Introduction}

With the acceleration of global economic integration, market competition becomes among supply chains rather than among enterprises. Supply chain management turns into a hotspot in enterprise and research circles. The bullwhip effect (BWE), defined by Lee et. al., [1], indicates the amplification phenomenon of order quantity from downstream enterprises to upstream enterprises in a supply chain. They also proposed five possible incentives, including price fluctuation and promotion, batched order, rationing game, demand signal processing, and non-zero leadtime. BWE exists widely in many industries. But BWE has many drawbacks to a supply chain, including inventory backlog, cost raise, production misleading, etc., BWE is so important that it becomes one core domain in supply chain management.

Over the years, many scholars and entrepreneurs study BWE constantly. Influence factor and reduction approach are two main research aspects. Croson et. al., [2] analyzed a multi-echelon supply chain. They stated that point of sale data sharing could reduce BWE. Zhang [3] studied the impact of three popular forecasting methods in a simple twoechelon supply chain. When demand was an AR (1) process and replenishment policy was order-up-to level, the forecast error variance remained unchanged under each method, including moving average, exponential smoothing and minimum mean-squared error. Ma et. al., [4] extended the research of Zhang with the same theory. They analyzed the three methods in a supply chain including one supplier and two retailers. Considering fuzzy

*Corresponding Author 
demand, leadtime and order size, Zarandi et. al., [5] developed an agent-based model to relieve BWE and lower gross cost.

These researchers above mainly uses mathematical derivation or simulation model to conduct research. They frequently assumes that demand meets a certain stationary distribution. But trend demand during a period of time abounds in real environments. Chen et. al., [6] studied the impact of trend demand for BWE early. Concerning two categories of demand, i.e., autocorrelation stationary demand and linear trend demand, they compared simple exponential smoothing forecasting method with double exponential smoothing forecasting method. They found that BWE was closely linked to leadtime and smoothing parameters for both categories. Enterprises should compress leadtime and choose small smoothing parameters to reduce BWE. Trend size had few effects on BWE for linear trend demand. Moreover, double exponential smoothing could bring a heavier order fluctuation compared with simple exponential smoothing. However, the research had some limitations. They mainly analyzed the lower bound of BWE in a two-echelon supply chain without considering safety stock. The smoothing parameters used in both methods were constants, which did not change in a long time and not tally with an actual situation. Additionally, the forecast error variance in order-up-to level policy was estimated without mathematical derivation.

On the basis of Chen et. al.,'s research, Bayraktar et. al., [7] simulated a two-echelon supply chain using Excel software. The demand in their study had seasonal linear trend, and they used Holt-Winters three-parameter forecasting method. They argued that different smoothing parameters had different influence on BWE. Season smoothing parameter had a smaller impact compared with level or trend smoothing parameter. The seasonal change of demand might be beneficial for BWE reduction. But they ignored the ability of choosing smoothing parameters dynamically of an enterprise. They also did not describe the detailed processes about solving and handling forecast error variance in order-up-to level policy. Wright et. al., [8] simulated a multi-echelon supply chain with local linear trend demand using four forecasting methods. They figured that Holt twoparameter method and Brown's single-parameter method could reduce BWE compared with simple exponential smoothing and simple moving average. They used anchoring and adjustment replenishment policy, which meant the order-up-to level remained unchanged. Ordering with economic order quantity, Chaharsooghi et. al., [9] analyzed the impact of three forecasting methods. These methods were simple moving average, simple exponential smoothing, and autoregressive integrated moving average model. They stated that for seasonal linear trend demand, an accurate demand estimation would not always relieve BWE. Zhao et. al., [10] programmed a $\mathrm{C}++$ model to simulate a supply chain including one suppliers and some retailers. Through analysis of variance, they identified the characteristics of different methods and the value of information sharing. Najafi et. al., [11] also researched linear trend demand. They found that BWE generated by forecasting method from small to big are the one by linear regression forecasting, by double exponential smoothing, and by double moving average. But their replenishment policy was plain. Order amount was calculated by the gap between forecasted demand and current inventory. Shipment delay and safety stock were out of consideration. Combining dampen Holt forecasting method with control theory, Li et. al., [12] analyzed the stable criterion of supply chain from the view of smoothing parameters. Through the BWE transformation of frequency domain, they argued that dampen Holt forecasting method sometimes might lead to BWE, but sometimes might not.

Generally, not too much research studies BWE with trend demand. This paper continues to analyze this problem. This paper focuses on linear trend demand, Holt twoparameter forecasting method and order-up-to level policy. Many enterprises use these methods and policies in reality. Since agent-based modeling is an efficient and effective technique to analyze complex systems including supply chain, an agent-based model is built in this study. This model has some other important characteristics. This model 
simulates a multi-echelon supply chain, which can reveal some complex patterns of the whole supply chain. In order to tally with practical situation, enterprises in the supply chain dynamically and intelligently fit their demand distributions and estimate relevant parameters. The fluctuation of BWE itself, which is represented by the standard deviation of BWE, is also analyzed.

The remainder of this paper is organized as follows. Section 2 describes the actions in a supply chain and builds a four-echelon agent-based simulation model. Section 3 conducts simulation and discusses BWE from four aspects. Section 4 draws some conclusions.

\section{Model Building}

This section describes and builds a four-echelon supply chain, which is a common multi-echelon model [13]. The supply chain contains six roles, i.e., an end customer, a retailer, a wholesaler, a distributor, a manufacturer and an out supplier. All players sell homogeneous products. Figure 1, displays all the actions in the supply chain at every simulation tick.

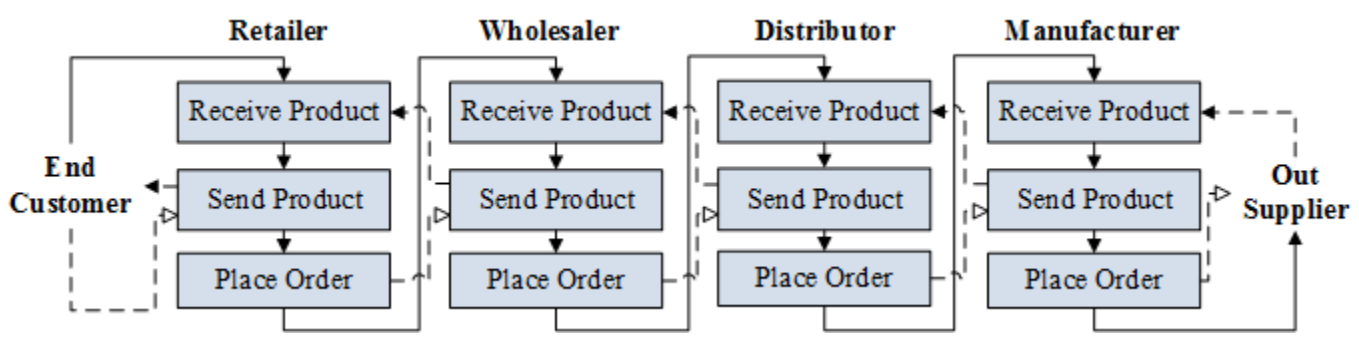

Figure 1. Player Action at Every Simulation Tick

\subsection{Model Description}

The solid lines with solid arrows in Figure 1 describe the action sequence. The end customer is the demand source and driven element. Its demand for the retailer has a linear trend and can be depicted as:

$$
\begin{aligned}
& R_{t}=l+b t+r_{t}, \\
& r_{t}=\rho r_{t-1}+e_{t} .
\end{aligned}
$$

$R_{t}$ means the end customer demand or requirement at simulation tick $t . l$ is an initial demand level. $b$ is a trend size. $r_{t}$ is the noise of customer demand, and it is an AR(1) process with autocorrelation coefficient $\rho$ and random error $e_{t} .|\rho|<1$ assures the noise is stationary. $e_{t}$ is an i.i.d. normally distributed random error with mean 0 and variance $\tau^{2}$. Eq. (1) is similar to the assumptions in [6], except that a normally distributed noise is changed to an AR(1) noise. The reason is that an $\mathrm{AR}(1)$ process equals a normal distribution when $\rho=0$. Eq. (1) is also a common assumption in the field of trend time series analysis.

The main actions of every player at every tick are receiving product, sending product and placing order. The dashed lines with hollow arrows in Figure 1, represent information flows, while the dashed lines with solid arrows represent material flows. Information flows mean order information interactions, which are assumed with no time delay. Material flows mean product delivery interactions, which are assumed with pure time delay. Thus a replenishment leadtime $L$ exits between two adjacent players.

The first action is receiving product. Every player receives product from its adjacent upstream player. In-stock inventory $v_{t}$ and pipeline inventory $p_{t}$ are handled. Let $s_{t}^{+}$ denote the shipment from adjacent upstream player at tick $t$, then: 


$$
\begin{aligned}
& v_{t}=v_{t-1}+s_{t-L^{\prime}}^{+} \\
& p_{t}=p_{t-1}-s_{t-L}^{+}+s_{t-1}^{+} .
\end{aligned}
$$

The second action is sending product. Every player decides a shipment according to demand and inventory. Let $s_{t}$ and $d_{t}$ denote the shipment and demand of a player at tick $t$, then:

$$
\begin{aligned}
& s_{t}=\min \left(d_{t}, v_{t}\right), \\
& v_{t}=\max \left(v_{t}-d_{t}, 0\right) .
\end{aligned}
$$

The last action is placing order, which contains three procedures. Firstly, demand distribution and parameters are dynamically fitted using observed demand information. Secondly, the demand expectation and forecast error variance of leadtime are calculated. Thirdly, an order size is solved and passed to an adjacent upstream player.

Realizing a trend demand, every player supposes and believes that its demand $d_{t}$ fits Eq. (6) in a period of time according to Holt forecasting method [14]. $l_{t}$ is the demand level at tick $t$, and $b_{t}$ is the demand trend at tick $t$. $\varepsilon_{t}$ is an i.i.d. normally distributed random error at tick $t$ with mean 0 and variance $\sigma^{2} . \alpha$ and $\beta$ are two smoothing parameters. The forecasted demand of tick $t+k(k \geq 1)$ at tick $t$ is defined as Eq. (7).

$$
\begin{aligned}
& d_{t+1}=l_{t}+b_{t}+\varepsilon_{t+1} \\
& l_{t}=\alpha d_{t}+(1-\alpha)\left(l_{t-1}+b_{t-1}\right), \\
& b_{t}=\beta\left(l_{t}-l_{t-1}\right)+(1-\beta) b_{t-1} . \\
& \bar{d}_{t+k}=l_{t}+k b_{t}, k \geq 1
\end{aligned}
$$

Every player fits its demand distribution with observed demand $\left\{d_{t-n+1}, d_{t-n+2}, \ldots\right.$, $\left.d_{t}\right\}$ using Maximum Likelihood Estimate theory. Considering the applicability and advantages of linear regression [11], least square method was used to determine the initial variables $l_{t-n}$ and $b_{t-n}$ with observed demand data. $l_{t-n}$ is set to the intercept of regression equation, while $b_{t-n}$ is set to the slope of regression equation. The maximum likelihood function is given in [15]. Bermudez et al. [16] have proved that maximizing the log-likelihood function is equivalent to minimizing the one step ahead mean square error (MSE). The one step ahead MSE is a function of $\alpha$ and $\beta$ after iteration. Therefore, maximizing the log-likelihood function is equivalent to:

$$
\operatorname{Min} \sum_{i=1}^{n}\left(d_{t-n+i}-l_{t-n+i-1}-b_{t-n+i-1}\right)^{2} /(n-1)=f(\alpha, \beta),
$$

$$
\text { s. t. } 0 \leq \alpha \leq 1,0 \leq \beta \leq 1 \text {. }
$$

Every player applies L-BFGS-B algorithm [17] to solving this nonlinear programming problem as Eq. (8). Thus optimal parameters $\bar{\alpha}_{t}$ and $\bar{\beta}_{t}$ at tick $t$ are obtained. If the algorithm cannot find an optimal solution, $\bar{\alpha}_{t}$ and $\bar{\beta}_{t}$ are both set to 0 , which indicates a linear regression forecasting. After $\alpha$ and $\beta$ are estimated, $\sigma^{2}$ is estimated as:

$$
\bar{\sigma}_{t}^{2}=\sum_{i=1}^{n}\left(d_{t-n+i}-l_{t-n+i-1}-b_{t-n+i-1}\right)^{2} /(n-1) .
$$

Then every player uses Holt method to calculate the total expected demand $\bar{D}_{t}$ and forecast error variance $\bar{E}_{t}^{2}$ of replenishment leadtime $L$. They are given by: 


$$
\begin{aligned}
& \bar{D}_{t}=L l_{t}+\frac{L(L+1) b_{t}}{2}, \\
& \text { if } L=1 \text { then } \bar{E}_{t}^{2}=\bar{\sigma}_{t}^{2}, \\
& \text { if } L \geq 2 \text { then } \bar{E}_{t}^{2}=\bar{\sigma}_{t}^{2}\left\{1+\sum_{i=1}^{L-1}\left[1+\frac{i(i+1) \bar{\alpha}_{t} \bar{\beta}_{t}}{2}+i \bar{\alpha}_{t}\right]^{2}\right\}
\end{aligned}
$$

Proof. See Appendix A.

After forecasting, an order size could be solved according to order-up-to level policy. This policy is widely used in research and reality, and is the standard policy of many ERP or MRP softwares [18]. Order size is calculated as:

$$
q_{t}=\max \left(\bar{D}_{t}+z \bar{E}_{t}-v_{t}-p_{t}, 0\right), t>n \text {. }
$$

Eq. (12) is feasible only if tick $t$ is bigger than $n$ in the simulation, because a player needs the demand data of beginning $n$ times to fit an original distribution. $z$ is the z-score determined by a desired service level. Since a player believes that its demand accords with Eq. (6), the conditional cumulative density function of gross demand in replenishment leadtime could be regarded as the normal distribution function [3]. This function has a mean $\bar{D}_{t}$ and a standard deviation $\bar{E}_{t}$. Therefore, $z$ is the normal $\mathrm{z}$-score.

Every player uses Eq. (13) to determine an order size in the beginning $n$ ticks. This equation is estimated considering linear trend. It aims at warming up the simulation model quickly.

$$
q_{t}=d_{t}+L b, t \leq n
$$

Then order information is passed to the upstream. This order quantity becomes the demand of an adjacent upstream player. Let $d_{t}^{+}$denote the demand of an adjacent upstream player at tick $t$, then:

$d_{t}^{+}=q_{t^{*}}$

Thus a player finishes the placing order action. All the actions are finished by the outer supplier. Since the outer supplier is not a concerned object, its supply capability is assumed as infinite with a leadtime $L$. This is a common hypothesis in many studies [19].

\subsection{Model Implementation}

To conduct subsequent simulation, the model described above should be switched to a specific agent-based model. Repast is one of the most popular agent-based simulation platforms. Repast provides a series of advanced, free and open structure and runtime elements to help researchers to conveniently establish and run a complex adaptive system. It is widely used in supply chain, biomedicine, social science, etc. Procter \& Gamble also successfully applies it to managing and solving some challenging business problems [20]. The model in this paper is built on Repast Simphony and programmed with Java language. Programming approach is very flexible to build a complex model, because researchers can define any characteristics and interactions for agents during programming.

In this model, every player needs to fit demand distribution and estimate parameters. This sophisticated calculation part is implemented by $\mathrm{R}$. $\mathrm{R}$ is also one popular environment in data calculation field. One plug-in called rJava acts as the linkage between simulation and calculation. Simulation model combined Repast with R may have a broad application prospect, because the agent could be easily attached with intelligent and brilliant features using this method. This supply chain model is a typical example. 
After completing this model, 20 Java classes are established. The total number of code lines is 2279. To maintain the correctness of the model, massive testing procedures are used during the development. Almost every class function has a corresponding unit testing function. Every module has been examined with integration testing. Step debugging is also frequently conducted to inspect the data consistency between actual change and expected situation.

\section{Model Simulation and Analysis}

Some basic simulation parameters in the model are configured as Table 1. Parameters do not have measurement units, because the simulation results would not be affected by on units. All the parameters are explained in section 2. Notice that $\rho=0$ means that the noise accords with normal distribution. $z=1.6449$ means that desired service level is $95 \%$. The initial in-stock inventory $v_{0}$ is 1046 and the initial pipeline inventory $p_{0}$ is 0 . These two values can help to warm up the simulation model quickly, since $L \times l+z \times \sqrt{L \times \tau^{2}} \approx 1046$. All the values about product quantity in the following simulation are integers.

Table 1. Basic Simulation Parameter

\begin{tabular}{cl|cl|cl|cl}
\hline Parameter & Value & Parameter & Value & Parameter & Value & Parameter & Value \\
\hline$L$ & 2 & $l$ & 500 & $b$ & 4 & $\rho$ & 0 \\
$\tau^{2}$ & 400 & $R_{0}$ & 500 & $n$ & 30 & $z$ & 1.6449 \\
$v_{0}$ & 1046 & $p_{0}$ & 0 & $s_{0}$ & 0 & & \\
\hline
\end{tabular}

After inputting this parameters and conducting the simulation process, the order quantities of all players are shown in Figure 2. The letters "C, R, W, D, M" in the figure represent the end customer, the retailer, the wholesaler, the distributor and the manufacturer, respectively.

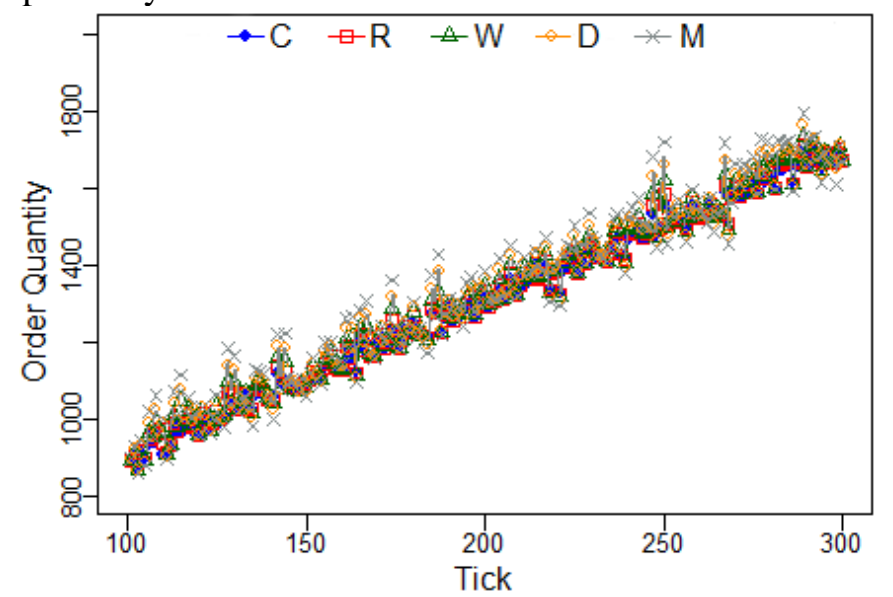

Figure 2. Order Quantity of Every Player

According to [21-[22], BWE can be measured by the ratio of player order quantity variance and customer demand variance, i.e.,

$$
B_{i}=\sum_{t=a}^{T}\left(q_{t, i}-\frac{1}{T-a+1} \sum_{t=a}^{T} q_{t, i}\right)^{2} / \sum_{t=a}^{T}\left(q_{t, 0}-\frac{1}{T-a+1} \sum_{t=a}^{T} q_{t, 0}\right)^{2} .
$$

$q_{t, i}$ is order quantity of different roles, and $i$ in $q_{t, i}$ represents the role. It could be $0,1,2$, 3 or 4 , which means the end customer, the retailer, the wholesaler, the distributor or the manufacturer. $T$ means the termination tick, which equals 300 here. The beginning $a-1$ 
ticks are excluded for calculation because of warming up process. $a$ equals 101 here. BWE of each player is 1.0016, 1.0080, 1.0198 and 1.0430, respectively. Outwardly, this supply chain does not have a significant BWE. But as shown in Figure 2, the order fluctuation of the manufacturer is significantly heavier than that of the end customer when removing the change of trend. This means Eq. (15) is not appropriate to calculate BWE when demand has a linear trend. In this study, BWE is measured by the ratio of the MSE of player order quantity and that of customer demand. MSE in the ratio is calculated by linear regression forecasting. Since the free degree of one-dimensional linear regression equals sample number minus two, BWE is:

$$
B_{i}=\frac{\sum_{t=a}^{T}\left(q_{t, i}-\bar{q}_{t, i}\right)^{2}}{t-a+1-2} / \frac{\sum_{t=a}^{T}\left(q_{t, 0}-\bar{q}_{t, 0}\right)^{2}}{t-a+1-2}=\sum_{t=a}^{T}\left(q_{t, i}-\bar{q}_{t, i}\right)^{2} / \sum_{t=a}^{T}\left(q_{t, 0}-\bar{q}_{t, 0}\right)^{2} \text {. (16) }
$$

$\bar{q}_{t, i}$ is a player's forecasted value of linear regression forecasting at tick $t$. Calculated by Eq. (16), BWE of each player is $1.5590,2.6469,4.6710$ and 8.2891, respectively. This indicates that the supply chain has a notable amplification of order fluctuation.

\subsection{Impact of Leadtime}

To analyze the impact of leadtime $L$, three rounds of simulations are conducted. $L$ is set to 2, 3 or 4 at each round on the basis of Table 1 . Initial in-stock inventory $v_{0}$ is also correspondingly set to 1046,1557 or 2066 . The model is simulated twenty times at each round. Then all players' BWE are calculated according to Eq. (16). Because of length limitation, detailed data is not displayed. The means and standard deviations of BWE are shown in Table 2.

Table 2. BWE of Different Leadtime

\begin{tabular}{|c|c|c|c|c|c|c|c|c|}
\hline \multirow{2}{*}{$\boldsymbol{L}$} & \multicolumn{4}{|c|}{ Mean of BWE } & \multicolumn{4}{|c|}{ Standard Deviation of BWE } \\
\hline & $\mathbf{R}$ & $\mathbf{W}$ & $\mathbf{D}$ & $\mathbf{M}$ & $\mathbf{R}$ & $\mathbf{W}$ & $\mathbf{D}$ & $\mathbf{M}$ \\
\hline 2 & 2.7830 & 9.1797 & 24.5273 & 65.6425 & 4.0056 & 18.7565 & 49.4813 & 113.3319 \\
\hline 3 & 4.0150 & 21.9056 & 223.6573 & 1130.3249 & 4.8640 & 37.1327 & 501.3043 & 1961.4727 \\
\hline 4 & 5.8975 & 53.5413 & 819.5778 & 16206.0021 & 6.2276 & 75.3432 & 1534.0704 & 30606.2480 \\
\hline
\end{tabular}

All means increase with the increase of $L$. One reason is that forecast error variance in replenishment leadtime increases with the increase of $L$. The means of upstream players are also bigger than that of downstream players. An interesting phenomenon is that the standard deviations also increase with the increase of $L$. This implies when leadtime is big, the fluctuation of BWE itself is also heavy. Notice that sometimes the means could be very big, especially for upstream players. This phenomenon is caused by a huge order fluctuation in a short time rather than an even big order fluctuation in a long time, e.g., Figure 3, shows a simulation result when $L=3$. The BWE in this figure from the retailer to the manufacturer is $2.8433,21.2386,313.8064$ and 2375.8389 . Therefore, the supply chain may become unstable in a period of time when leadtime is relatively big. 


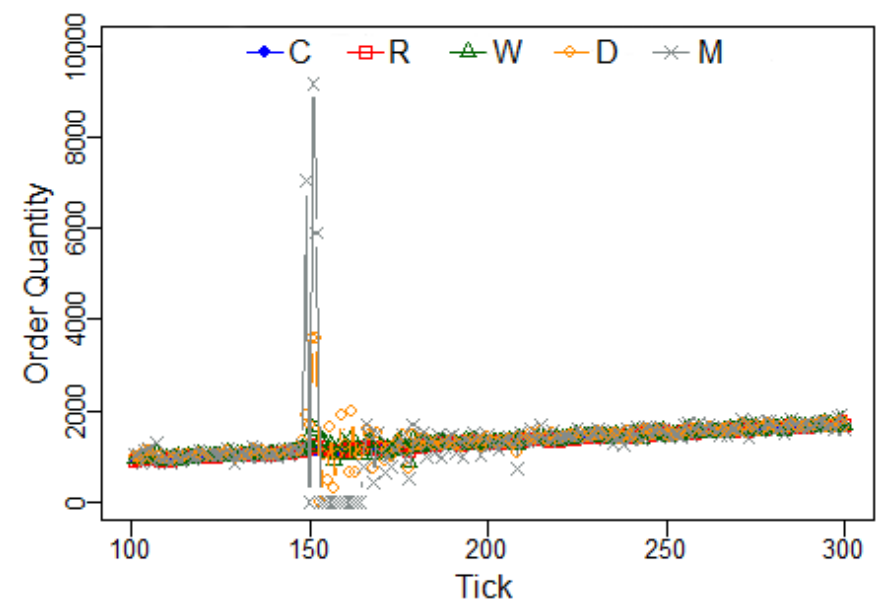

Figure 3. One Simulation Result when $L=3$

As shown in Figure 3, the means and the standard deviations both increase with the increase of $L$. Significance test is needed to verify whether leadtime affects BWE remarkably. T-test method is not suitable, because the data of some rounds are not in accordance with normal distribution. One nonparametric test method called "MannWhitney U test" is used instead. The order quantities of every player and every leadtime are regarded as independent samples. Two corresponding samples are successively chosen to form a test couple. The result is shown in Table 3. All of the p-values are smaller than 0.05 . Both BWE and its fluctuation aggravate with the increase of leadtime.

Table 3. Significance Test P-Value of Leadtime

\begin{tabular}{cclll}
\hline $\boldsymbol{L}$ Couple & \multicolumn{1}{c}{$\mathbf{R}$} & \multicolumn{1}{c}{ W } & \multicolumn{1}{c}{$\mathbf{D}$} & \multicolumn{1}{c}{$\mathbf{M}$} \\
\hline $\mathbf{2 ~ 3}$ & $5.8328 \mathrm{e}-06$ & $6.9092 \mathrm{e}-05$ & $5.2082 \mathrm{e}-05$ & $9.1048 \mathrm{e}-05$ \\
$\mathbf{2} \mathbf{4}$ & $1.3671 \mathrm{e}-06$ & $3.2864 \mathrm{e}-07$ & $2.8292 \mathrm{e}-09$ & $5.8036 \mathrm{e}-11$ \\
$\mathbf{3} \mathbf{4}$ & $2.1377 \mathrm{e}-05$ & 0.0003 & 0.0018 & 0.0005 \\
\hline
\end{tabular}

\subsection{Impact of Trend Size}

To analyze the impact of trend size $b$, three rounds of simulations are conducted. $b$ is set to 4,12 or 20 at each round on the basis of Table 1 . The model is also simulated twenty times at each round. Table 4 shows the means and standard deviations of BWE.

Table 4. BWE of Different Trend Size

\begin{tabular}{|c|c|c|c|c|c|c|c|c|}
\hline \multirow{2}{*}{$b$} & \multicolumn{4}{|c|}{ Mean of BWE } & \multicolumn{4}{|c|}{ Standard Deviation of BWE } \\
\hline & $\overline{\mathbf{R}}$ & $\mathbf{W}$ & D & $\mathbf{M}$ & $\mathbf{R}$ & $\mathbf{W}$ & D & $\mathbf{M}$ \\
\hline 4 & 2.7830 & 9.1797 & 24.5273 & 65.6425 & 4.0056 & 18.7565 & 49.4813 & 113.3319 \\
\hline 12 & 2.7837 & 9.1697 & 24.4345 & 66.0569 & 4.0054 & 18.7601 & 49.5183 & 116.9231 \\
\hline 20 & 2.7798 & 9.1603 & 24.7241 & 66.9963 & 4.0063 & 18.7609 & 49.4296 & 117.0437 \\
\hline
\end{tabular}

The means and standard deviations change very little with the increase of $b$. Table 5 shows the result of significance test. All of the p-values are bigger than 0.05. Therefore, trend size has little influence on BWE.

Table 5. Significance Test P-value of Trend Size

\begin{tabular}{clccc}
\hline $\boldsymbol{b}$ Couple & \multicolumn{1}{c}{ R } & W & D & M \\
\hline 4 12 & 0.9627 & 0.9094 & 0.8462 & 0.9307 \\
4 20 & 1 & 0.8883 & 0.9733 & 0.9946 \\
12 20 & 0.9625 & 0.9840 & 0.8883 & 0.9413 \\
\hline
\end{tabular}




\subsection{Impact of Noise Variance}

To analyze the impact of noise variance $\tau^{2}$, four rounds of simulations are conducted. $\tau^{2}$ is set to $400,3600,10000$ or $\mathrm{U}(400,10000)$ at each round on the basis of Table 1 . Here $\mathrm{U}(400,10000)$ means a uniform distribution with maximum value 10000 and minimum value 400 . Notice that the noise of end customer demand still accords with the normal distribution. The model is also simulated twenty times at each round. Table 6 shows the means and standard deviations of BWE.

Table 6. BWE of Different Noise Variance

\begin{tabular}{|c|c|c|c|c|c|c|c|c|}
\hline \multirow{2}{*}{$\tau^{2}$} & \multicolumn{4}{|c|}{ Mean of BWE } & \multicolumn{4}{|c|}{ Standard Deviation of BWE } \\
\hline & $\mathbf{R}$ & $\mathbf{W}$ & D & $\mathbf{M}$ & $\mathbf{R}$ & $\mathbf{W}$ & $\mathbf{D}$ & $\mathbf{M}$ \\
\hline 400 & 2.7830 & 9.1797 & 24.5273 & 65.6425 & 4.0056 & 18.7565 & 49.4813 & 113.3319 \\
\hline 3600 & 1.8183 & 4.2046 & 11.8232 & 33.3269 & 0.5539 & 2.4977 & 10.3268 & 38.1420 \\
\hline 10000 & 1.6943 & 3.6826 & 10.1099 & 25.4038 & 0.2121 & 1.4297 & 8.7221 & 32.8679 \\
\hline $\mathrm{U}(400,10000)$ & 1.7327 & 3.7218 & 8.3653 & 16.2603 & 0.4024 & 1.7646 & 5.3121 & 10.4777 \\
\hline
\end{tabular}

Both the means and the standard deviations decrease with the increase of $\tau^{2}$. When $\tau^{2}$ accords with uniform distribution, the mean and the standard deviation are both smaller than that of three fixed $\tau^{2}$. Detailed analysis is presented below.

Figure 4, shows the means of BWE for the manufacturer under different $\tau^{2}$. Run numbers are arranged for convenient display. The simulation random seeds under four conditions for one run are the same. The means are relatively small and close to each other for the first fifteen runs. The pattern of means for the last five runs is a little complicated. All the means are relatively big when $\tau^{2}=400$. Meanwhile, when $\tau^{2}=3600$ or $\tau^{2}=10000$, the means are close to that when $\tau^{2}=400$ for the sixteenth, seventeenth or nineteenth run. But when $\tau^{2}=3600$ or $\tau^{2}=10000$, the means are significantly smaller than that when $\tau^{2}=400$ for the eighteenth or twentieth run. When $\tau^{2}$ accords with uniform distribution, the means are relatively small for the last five runs.

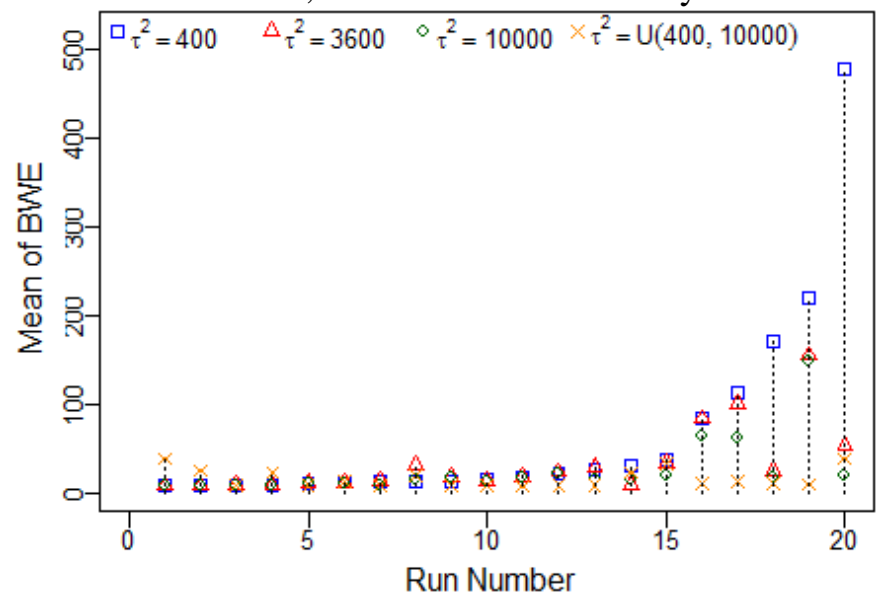

Figure 4. Mean of BWE for the Manufacturer

Figure 5, shows the order quantities for the twentieth run under different $\tau^{2}$. 


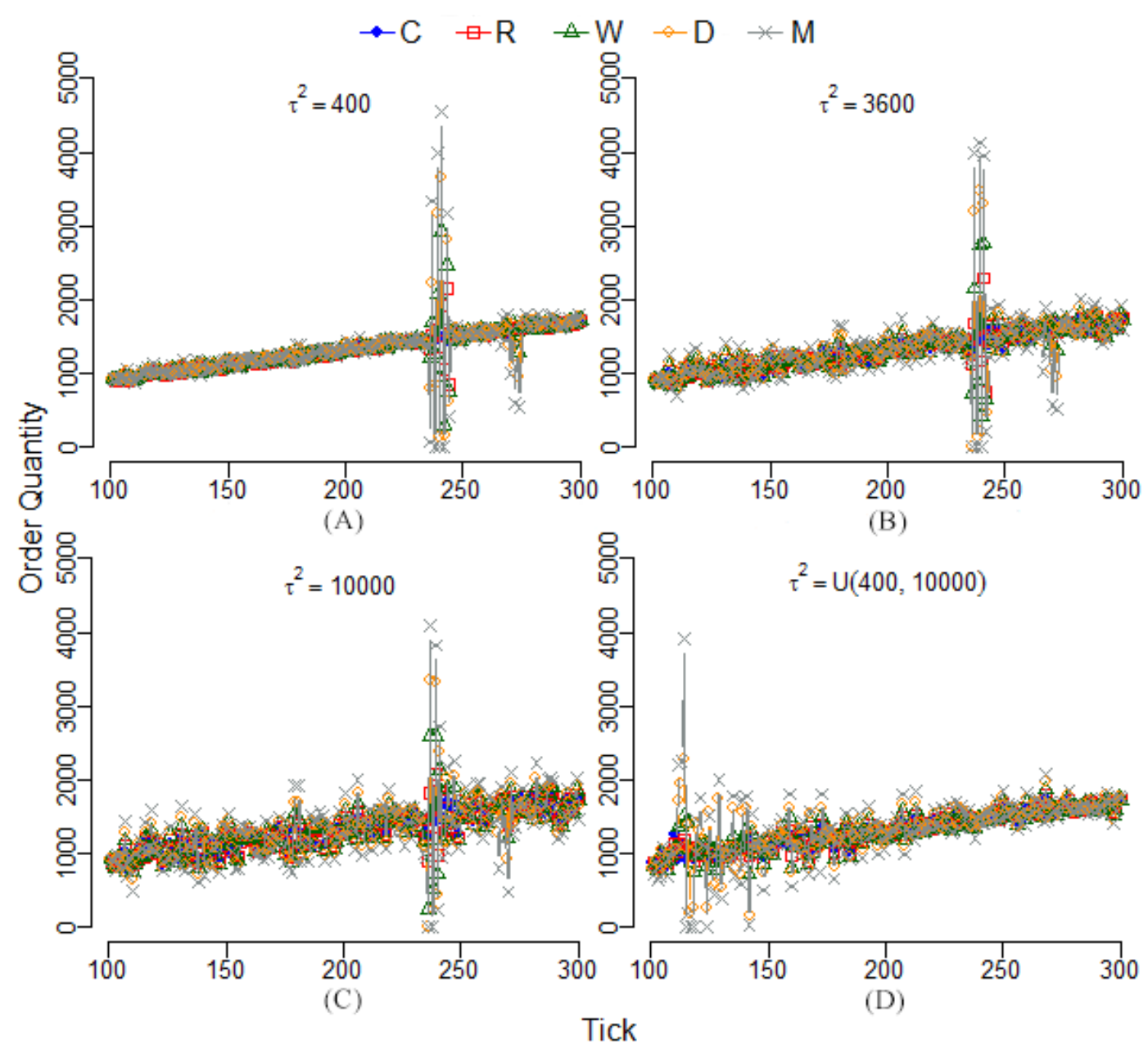

Figure 5. Order Quantity Comparison for the Twentieth Run

The patterns of order quantity are similar to each other when $\tau^{2}$ is fixed. A big mean of BWE is caused by a huge order fluctuation in a short time rather than an even big order fluctuation in a long time. If calculated with the stationary data, i.e., order quantities from tick 101 to tick 200, BWE of all players are shown in Table 7. They have few differences for different $\tau^{2}$. The amplification of order fluctuation from the downstream to the upstream is not as distinct as what is shown in Table 6 . When $\tau^{2}$ accords with uniform distribution, order quantity pattern is different. The order quantity fluctuation is relatively even for the whole period. This is the reason for that the mean and the standard deviation are both smaller than that of three fixed $\tau^{2}$ in Table 6 .

Table 7. BWE from Tick 101 to Tick 200 for the Twentieth Run

\begin{tabular}{ccccc}
\hline $\boldsymbol{\tau}^{2}$ & $\mathbf{R}$ & $\mathbf{W}$ & $\mathbf{D}$ & $\mathbf{M}$ \\
\hline $\mathbf{4 0 0}$ & 1.5933 & 2.6886 & 4.7210 & 8.3436 \\
$\mathbf{3 6 0 0}$ & 1.5942 & 2.6754 & 4.6785 & 8.2674 \\
$\mathbf{1 0 0 0 0}$ & 1.5958 & 2.6844 & 4.7089 & 8.3408 \\
\hline
\end{tabular}

Through the discussion above, some conclusions have been found in this supply chain. If the noise variance is fixed during a period of time, the order quantity fluctuation is even during most of the time. But this fluctuation may be violent in a short time, which would affect the whole BWE. Meanwhile, a small noise variance may cause a big BWE. When 
noise variance accords with uniform distribution, a violent order fluctuation during a period of time would have a low probability of appearance.

Table 8 shows the result of significance test. All of the p-values are bigger than 0.05 . Therefore, noise variance does not affect BWE significantly, even though noise variance randomly changes in accordance with uniform distribution. This conclusion matches the analysis above.

Table 8. Significance Test P-Value of Noise Variance

\begin{tabular}{ccccc}
\hline $\boldsymbol{\tau}^{2}$ Couple & $\mathbf{R}$ & $\mathbf{W}$ & $\mathbf{D}$ & $\mathbf{M}$ \\
\hline $\mathbf{4 0 0} \mathbf{3 6 0 0}$ & 0.9680 & 0.7584 & 0.7584 & 0.6980 \\
$\mathbf{4 0 0} \mathbf{1 0 0 0 0}$ & 0.9197 & 0.6395 & 0.4777 & 0.3547 \\
$\mathbf{4 0 0} \sim \mathbf{U}(\mathbf{4 0 0 ,} \mathbf{1 0 0 0 0 )}$ & 0.6783 & 0.3408 & 0.2315 & 0.0634 \\
$\mathbf{3 6 0 0} \sim \mathbf{1 0 0 0 0}$ & 0.8985 & 0.9042 & 0.7381 & 0.4291 \\
$\mathbf{3 6 0 0} \sim \mathbf{U ( 4 0 0 , 1 0 0 0 0 )}$ & 0.6017 & 0.4612 & 0.2315 & 0.0859 \\
$\mathbf{1 0 0 0 0} \sim \mathbf{U ( 4 0 0 , 1 0 0 0 0 )}$ & 0.7529 & 0.5648 & 0.5468 & 0.3273 \\
\hline
\end{tabular}

\subsection{Impact of Noise Autocorrelation Coefficient}

To analyze the impact of noise autocorrelation coefficient $\rho$, nine rounds of simulations are conducted. Notice that the noise of end customer demand is an AR(1) process now. At each round, $\rho$ is set to $-0.9,-0.75,-0.5,-0.25,0,0.25,0.5,0.75$ or 0.9 on the basis of Table 1. Random error variance $\tau^{2}$ is also changed with different $\rho$. It is given by $\tau^{2}=400\left(1-\rho^{2}\right)$. This measure guarantees the noise variance remain unchanged for different $\rho$. The model is simulated twenty times at each round. The means and standard deviations of all players' BWE are calculated according to Eq. (16). Then two corresponding samples with adjacent $\rho$ are successively chosen to participate in a Mann-Whitney U test. Figure 6, shows the impact of noise autocorrelation coefficient for the mean of BWE. The solid line indicates the difference between two adjacent means is insignificant, while the dashed line indicates significant. The enlarged part whose $\rho$ ranges from -0.90 to -0.25 is shown in the small box.

Generally, the means of downstream players are always smaller than that of upstream players at the same $\rho$. The means of all players roughly change slowly at first, then increase significantly with a big range, and then decrease. When $\rho$ is around 0.5 , the amplifications of order quantity fluctuation are particularly notable for upstream players.

For the retailer, the mean has two significant downtrends when $\rho$ increases from -0.5 to -0.25 and from 0.75 to 0.9 . The mean has two significant uptrends when $\rho$ increases from 0 to 0.5 . The differences of means between other two adjacent values are insignificant. For the wholesaler, when $\rho<-0.25$, the differences of means at these segments are insignificant. When $\rho>-0.25$, the mean has two significant uptrends at first, then no significant difference, and then a significant decrease. For the distributor and the manufacturer, when $\rho<-0.25$, the differences of means at these segments are insignificant. When $\rho>-0.25$, the means have three significant uptrends with big scopes at first, then no significant difference. 


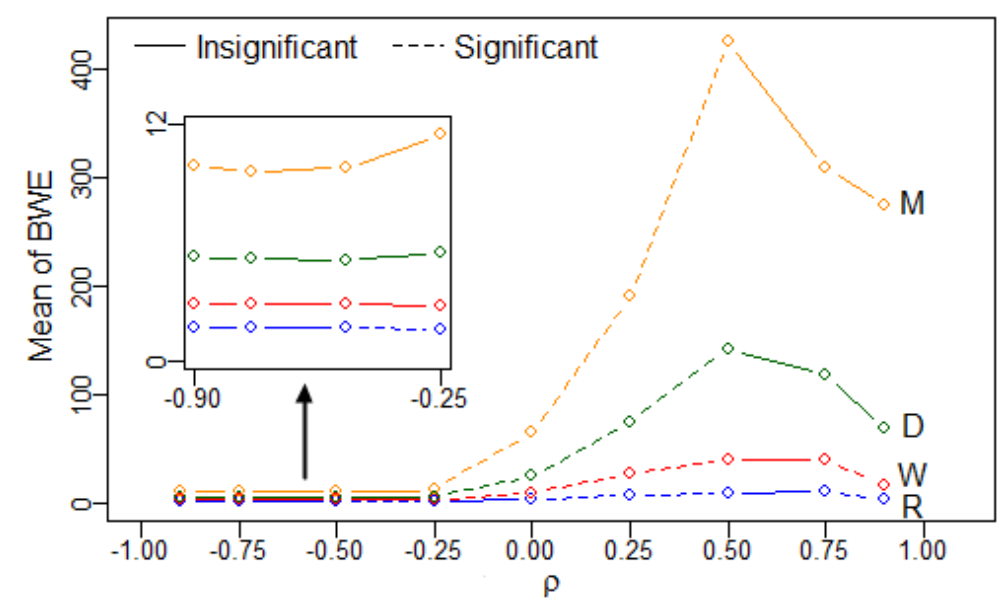

Figure 6. Impact of Noise Autocorrelation Coefficient for Mean

Figure 7, shows the standard deviations of BWE. The standard deviations of downstream players are always smaller than that of upstream players at the same $\rho$. The change pattern of standard deviation is similar to that of mean. Therefore, Pearson correlation coefficients between standard deviation and mean are calculated. They are $0.9187,0.9432,0.9755$ and 0.9218 , and p-values are $0.0005,0.0001,7.45 \mathrm{e}-06$ and 0.0004 . The change processes of standard deviation and mean for all players have very strong correlations.

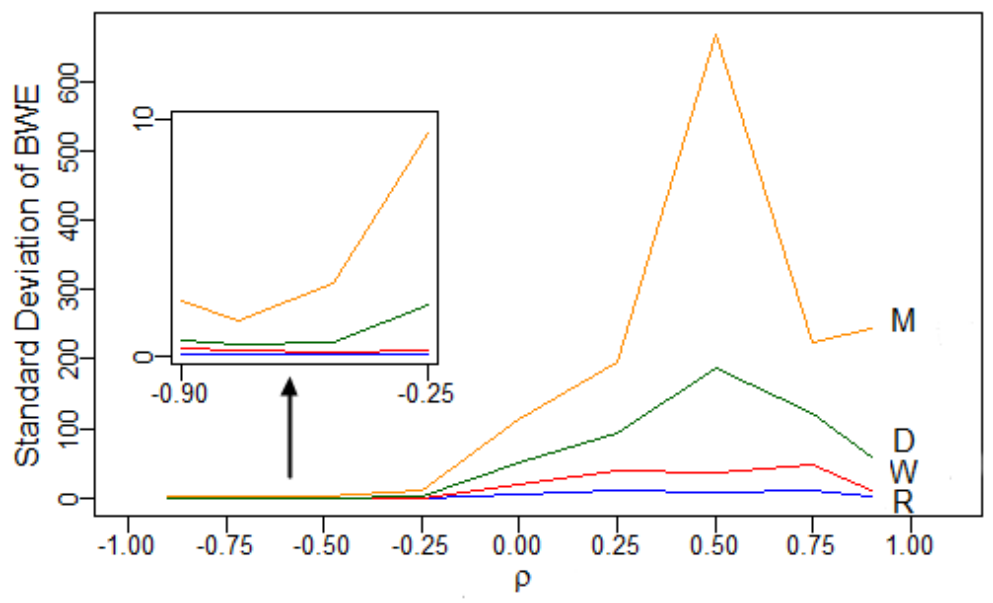

Figure 7. Impact of Noise Autocorrelation Coefficient for Standard Deviation

\section{Conclusion}

This paper studies BWE in a multi-echelon supply chain with linear trend demand. An agent-based simulation model is built using Repast and R. One highlight is that the players in the model do not need to know their accurate demand distributions beforehand. They dynamically and intelligently fit the distributions using recent observed demand information. Then they use Holt two-parameter forecasting method and order-up-to level replenishment policy to solve order quantities. During the analysis, this paper explains the limitation of classic BWE measurement for linear trend demand and presents a more appropriate one.

The results show that BWE and the fluctuation of it both aggravate with the increase of leadtime. Furthermore, order fluctuation may be easy to be violent in a short time if leadtime is big. Hence, enterprises should take measures to compress leadtime for BWE reduction. The trend size of customer demand has little influence on BWE. These two 
rules are similar to the results in the research of some scholars. But this study is based on a different forecasting method and a different BWE measurement.

When the noise variance of end customer demand accords with normal distribution, it does not affect BWE significantly. If the noise variance is fixed, the order quantity fluctuation is even during most of the time. But a violent fluctuation in a short time may appear. A small noise variance may cause a big BWE. When noise variance randomly changes with uniform distribution, a violent fluctuation during a period of time would have a low probability of appearance. When the noise is an AR(1) process, the means of all players roughly change slowly at first, then increase significantly with a big range, and then decrease. Meanwhile, the change processes of mean and standard deviation have very strong correlations. It is also found that the BWE change rule of a retailer is very different from that of an upstream player. Therefore, research on multi-echelon supply chain is meaningful.

These conclusions could help to analyze and reduce BWE when demand is characterized by trend. Some further research could be extended. This agent-based model can be developed to analyze BWE from some other aspects, including the impact of HoltWinters forecasting method and the impact of multiple players.

\section{Appendix A. Proof of Eq. (10) and Eq. (11)}

According to Holt method, expected demand $\bar{D}_{t}$ of leadtime $L$ can be calculated as:

$$
\bar{D}_{t}=\sum_{j=1}^{L} \bar{d}_{t+j}=\left(l_{t}+b_{t}\right)+\left(l_{t}+2 b_{t}\right)+\cdots+\left(l_{t}+L b_{t}\right)=L l_{t}+\frac{L(L+1) b_{t}}{2} .
$$

$\alpha$ and $\beta$ are estimated as $\bar{\alpha}_{t}$ and $\bar{\beta}_{t}$. Then $l_{t}$ and $b_{t}$ in Eq. (6) could be represented by:

$$
\begin{aligned}
l_{t} & =\bar{\alpha}_{t} d_{t}+\left(1-\bar{\alpha}_{t}\right)\left(l_{t-1}+b_{t-1}\right)=\bar{\alpha}_{t}\left(l_{t-1}+b_{t-1}+\varepsilon_{t}\right)+\left(1-\bar{\alpha}_{t}\right)\left(l_{t-1}+b_{t-1}\right) \\
& =l_{t-1}+b_{t-1}+\bar{\alpha}_{t} \varepsilon_{t} \\
b_{t} & =\bar{\beta}_{t}\left(l_{t}-l_{t-1}\right)+\left(1-\bar{\beta}_{t}\right) b_{t-1}=\bar{\beta}_{t}\left(l_{t-1}+b_{t-1}+\bar{\alpha}_{t} \varepsilon_{t}-l_{t-1}\right)+\left(1-\bar{\beta}_{t}\right) b_{t-1} \\
& =b_{t-1}+\bar{\alpha}_{t} \bar{\beta}_{t} \varepsilon_{t}
\end{aligned}
$$

The believable trend and level of actual demand of tick $t+k(k \geq 1)$ are:

$$
\begin{aligned}
& \boldsymbol{k}=1: b_{t+k-1}=b_{t,} \\
& \boldsymbol{k} \geq 2: b_{t+k-1}=b_{t+k-2}+\bar{\alpha}_{t} \bar{\beta}_{t} \varepsilon_{t+k-1}=b_{t+k-3}+\bar{\alpha}_{t} \bar{\beta}_{t} \varepsilon_{t+k-2}+\bar{\alpha}_{t} \bar{\beta}_{t} \varepsilon_{t+k-1}=\ldots \\
&=b_{t}+\bar{\alpha}_{t} \bar{\beta}_{t} \sum_{i=1}^{k-1} \varepsilon_{t+i} \\
& \boldsymbol{k}=1: l_{t+k-1}=l_{t} \\
& \boldsymbol{k} \geq 2: l_{t+k-1}=l_{t+k-2}+b_{t+k-2}+\bar{\alpha}_{t} \varepsilon_{t+k-1}=l_{t+k-3}+b_{t+k-3}+\bar{\alpha}_{t} \varepsilon_{t+k-2} \\
&+b_{t+k-2}+\bar{\alpha}_{t} \varepsilon_{t+k-1}=\ldots=l_{t}+\left(b_{t}+b_{t+1}+\cdots+b_{t+k-2}\right)+\bar{\alpha}_{t}\left(\varepsilon_{t+1}+\varepsilon_{t+2}\right. \\
&\left.+\cdots+\varepsilon_{t+k-1}\right)=l_{t}+b_{t}+\left(b_{t}+\bar{\alpha}_{t} \bar{\beta}_{t} \varepsilon_{t+1}\right)+\cdots+\left(b_{t}+\bar{\alpha}_{t} \bar{\beta}_{t} \varepsilon_{t+1}+\ldots \ldots\right. \\
&\left.+\bar{\alpha}_{t} \bar{\beta}_{t} \varepsilon_{t+k-2}\right)+\bar{\alpha}_{t}\left(\varepsilon_{t+1}+\varepsilon_{t+2}+\cdots+\varepsilon_{t+k-1}\right)
\end{aligned}
$$




$$
=l_{t}+(k-1) b_{t}+\sum_{i=1}^{k-1}\left[(k-1-i) \bar{\alpha}_{t} \bar{\beta}_{t}+\bar{\alpha}_{t}\right] \varepsilon_{t+i}
$$

Then, the actual demand of tick $t+k(k \geq 1)$ is:

$$
\begin{aligned}
& \boldsymbol{k}=1: d_{t+k}=l_{t}+b_{t}+\varepsilon_{t+1}, \\
& \boldsymbol{k} \geq 2: d_{t+k}=l_{t+k-1}+b_{t+k-1}+\varepsilon_{t+k}=b_{t}+\bar{\alpha}_{t} \bar{\beta}_{t} \sum_{i=1}^{k-1} \varepsilon_{t+i} \\
& \\
&+l_{t}+(k-1) b_{t}+\sum_{i=1}^{k-1}\left[(k-1-i) \bar{\alpha}_{t} \bar{\beta}_{t}+\bar{\alpha}_{t}\right] \varepsilon_{t+i}+\varepsilon_{t+k} \\
&=l_{t}+k b_{t}+\varepsilon_{t+k}+\sum_{i=1}^{k-1}\left[(k-i) \bar{\alpha}_{t} \bar{\beta}_{t}+\bar{\alpha}_{t}\right] \varepsilon_{t+i} .
\end{aligned}
$$

According to Holt method, the forecasted demand of tick $t+k(k \geq 1)$ is: $\bar{d}_{t+k}=l_{t}+k b_{t}$.

Then, the forecast error of the total demand of leadtime $L$ at tick $t$ is:

$$
\begin{aligned}
\boldsymbol{L} & =1: \bar{E}_{t}=D_{t}-\bar{D}_{t}=d_{t+1}-\bar{d}_{t+1}=b_{t}+l_{t}+\varepsilon_{t+1}-\left(b_{t}+l_{t}\right)=\varepsilon_{t+1}, \\
\boldsymbol{L} & \geq 2: \bar{E}_{t}=D_{t}-\bar{D}_{t}=\sum_{j=1}^{L} d_{t+j}-\sum_{j=1}^{L} \bar{d}_{t+j}=\sum_{j=1}^{L}\left(d_{t+j}-\bar{d}_{t+j}\right)=\varepsilon_{t+1} \\
& +\sum_{j=2}^{L}\left(d_{t+j}-\bar{d}_{t+j}\right)=\varepsilon_{t+1}+\sum_{j=2}^{L}\left(\varepsilon_{t+j}+\sum_{i=1}^{j-1}\left[(j-i) \bar{\alpha}_{t} \bar{\beta}_{t}+\bar{\alpha}_{t}\right] \varepsilon_{t+i}\right) \\
& =\left[1+\sum_{j=1}^{L-1}\left(j \bar{\alpha}_{t} \bar{\beta}_{t}+\bar{\alpha}_{t}\right)\right] \varepsilon_{t+1}+\left[1+\sum_{j=1}^{L-2}\left(j \bar{\alpha}_{t} \bar{\beta}_{t}+\bar{\alpha}_{t}\right)\right] \varepsilon_{t+2}+\cdots \\
& +\left[1+\left(\bar{\alpha}_{t} \bar{\beta}_{t}+\bar{\alpha}_{t}\right)\right] \varepsilon_{t+L-1}+\varepsilon_{t+L} .
\end{aligned}
$$

$\sigma^{2}$ is estimated as $\bar{\sigma}_{t}^{2}$. Then the forecast error variance $\bar{E}_{t}^{2}$ at tick $t$ is:

$$
\begin{aligned}
L & =1: \bar{E}_{t}^{2}=\operatorname{Var}\left(D_{t}-\bar{D}_{t}\right)=\operatorname{Var}\left(\varepsilon_{t+1}\right)=\bar{\sigma}_{t}^{2} \\
L & \geq 2: \bar{E}_{t}^{2}=\operatorname{Var}\left(D_{t}-\bar{D}_{t}\right)=\operatorname{Var}\left(\left[1+\sum_{j=1}^{L-1}\left(j \bar{\alpha}_{t} \bar{\beta}_{t}+\bar{\alpha}_{t}\right)\right] \varepsilon_{t+1}\right. \\
& \left.+\left[1+\sum_{j=1}^{L-2}\left(j \bar{\alpha}_{t} \bar{\beta}_{t}+\bar{\alpha}_{t}\right)\right] \varepsilon_{t+2}+\cdots+\left[1+\left(\bar{\alpha}_{t} \bar{\beta}_{t}+\bar{\alpha}_{t}\right)\right] \varepsilon_{t+L-1}+\varepsilon_{t+L}\right) \\
& =\bar{\sigma}_{t}^{2}\left\{\left[1+\frac{(L-1) L \bar{\alpha}_{t} \bar{\beta}_{t}}{2}+(L-1) \bar{\alpha}_{t}\right]^{2}\right.
\end{aligned}
$$




$$
\begin{aligned}
& \left.+\left[1+\frac{(L-2)(L-1) \bar{\alpha}_{t} \bar{\beta}_{t}}{2}+(L-2) \bar{\alpha}_{t}\right]^{2}+\cdots+\left[1+\frac{1 \times 2 \bar{\alpha}_{t} \bar{\beta}_{t}}{2}+\bar{\alpha}_{t}\right]^{2}+1\right\} \\
& =\bar{\sigma}_{t}^{2}\left\{1+\sum_{i=1}^{L-1}\left[1+\frac{i(i+1) \bar{\alpha}_{t} \bar{\beta}_{t}}{2}+i \bar{\alpha}_{t}\right]^{2}\right\}
\end{aligned}
$$

\section{Acknowledgments}

This work is supported by the National Natural Science Foundation of China (No. 71101005).

\section{References}

[1] H. L. Lee, V. Padmanabhan and S. Whang, "Information distortion in a supply chain: The bullwhip effect”, Management Science, vol. 43, no. 4, (1997), pp. 546-558.

[2] R. Croson and K. Donohue, "Impact of POS data sharing on supply chain management: An experimental study", Production and Operations Management, vol. 12, no. 1, (2003), pp. 1-11.

[3] X. Zhang, "The impact of forecasting methods on the bullwhip effect", International Journal of Production Economics, vol. 88, no. 1, (2004), pp. 15-27.

[4] J. Ma, B. Bao and X. Ma, "Inherent complexity research on the bullwhip effect in supply chains with two retailers: The impact of three forecasting methods considering market share", Abstract and Applied Analysis, vol. 2014, (2014), pp. 1-13.

[5] M. H. Zarandi, M. Pourakbar and I. B. Turksen, "A fuzzy agent-based model for reduction of bullwhip effect in supply chain systems", Expert Systems with Applications, vol. 34, no. 3, (2008), pp. 1680-1691.

[6] F. Chen, J. K. Ryan and D. S. Levi, "The impact of exponential smoothing forecasts on the bullwhip effect", Naval Research Logistics, vol. 47, no. 4, (2002), pp. 269-286.

[7] E. Bayraktar, S. C. L. Koh, A. Gunasekaran, K. Sari and E. Tatoglu, "The role of forecasting on bullwhip effect for E-SCM applications", International Journal of Production Economics, vol. 113, no. 1, (2008), pp. 193-204.

[8] D. Wright and X. Yuan, "Mitigating the bullwhip effect by ordering policies and forecasting methods", International Journal of Production Economics, vol. 113, no. 2, (2008), pp. 587-597.

[9] S. K. Chaharsooghi, H. Faramarzi and J. Heydari, "A simulation study on the impact of forecasting methods on the bullwhip effect in the supply chain", Proceedings of the IEEE International Conference on Industrial Engineering and Engineering Management, (2008), pp. 1875-1879.

[10] X. D. Zhao, J. X. Xie and J. Leung, "The impact of forecasting model selection on the value of information sharing in a supply chain", European Journal of Operational Research, vol. 142, no. 2, (2002), pp. 321-344.

[11] M. Najafi and R. Z. Farahani, "New forecasting insights on the bullwhip effect in a supply chain", IMA Journal of Management Mathematics, vol. 25, no. 3, (2014), pp. 259-286.

[12] Q. Y. Li, S. M. Disney and G. Gaalman, "Avoiding the bullwhip effect using damped trend forecasting and the order-up-to replenishment policy", International Journal of Production Economics, vol. 149, (2014), pp. 3-16.

[13] P. Alizadeh, "The role of forecasting parameters in reducing bullwhip effect", Advances in Production Engineering and Management, vol. 7, no. 3, (2012), pp. 177-186.

[14] C. Holt, "Forecasting trends and seasonals by exponentially weighted moving averages", ONR Memorandum, vol. 52, (1957).

[15] J. K. Ord, A. B. Koehler and R. D. Snyder, "Estimation and prediction for a class of dynamic nonlinear statistical models", Journal of the American Statistical Association, vol. 92, no. 440, (1997), pp. 16211629.

[16] J. D. Bermudez, J. V. Segura and E. Vercher, "Holt-Winters forecasting: An alternative formulation applied to UK air passenger data", Journal of Applied Statistics, vol. 34, no. 9, (2007), pp. 1075-1090.

[17] R. H. Byrd, P. Lu, J. Nocedal and C. Zhu, "A limited memory algorithm for bound constrained optimization", SIAM Journal on Scientific Computing, vol. 16, no. 5, (1995), pp. 1190-1208.

[18] K. Gilbert, "An ARIMA supply chain model", Management Science, vol. 51, no. 2, (2005), pp. 305-310.

[19] Y. Zhang and S. Bhattacharyya, "Analysis of B2B e-marketplaces: an operations perspective", Information Systems and E-Business Management, vol. 8, no. 3, (2010), pp. 235-256.

[20] M. J. North, C. M. Macal, J. S. Aubin, P. Thimmapuram, M. Bragen, J. Hahn, J. Karr, N. Brigham, M. E. Lacy and D. Hampton, "Multiscale agent-based consumer market modeling", Complexity, vol. 15, no. 5, (2010), pp. 37-47. 
[21] F. Chen, Z. Drezner, J. K. Ryan and D. S. Levi, "Quantifying the bullwhip effect in a simple supply chain: The impact of forecasting, lead times, and information", Management Science, vol. 46, no. 3, (2000), pp. 436-443.

[22] J. Costas, B. Ponte, D. de la Fuente, R. Pino and J. Puche, "Applying Goldratt's theory of constraints to reduce the bullwhip effect through agent-based modeling", Expert Systems with Applications, vol. 42, no. 4, (2015), pp. 2049-2060.

\section{Authors}

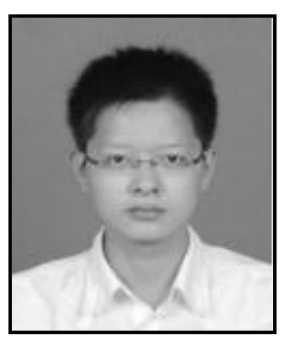

Hongsen Wang, he has received the Bachelor degree of Management in School of Management and Economics, Beijing Institute of Technology in 2010, Beijing, China. His subject is information management and information systems. He is now a Ph.D. candidate in School of Economics and Management, Beihang University, Beijing, China. His research areas are supply chain management, agent-based modeling and simulation, complex adaptive systems.

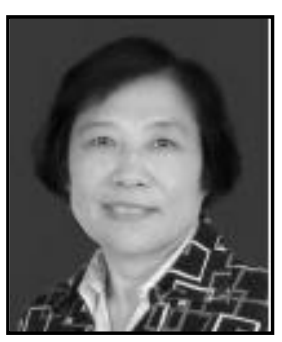

Suling Jia, she is a professor in School of Economics and Management, Beihang University, Beijing, China. Her research interests include supply chain management, management information system, IT project management, system dynamics and complex adaptive systems. She is also a standing director in Information System Branch of China Computer Users Association. 